$5,2 \mathrm{eV}$ bestimmt. Für die Dichte der festen Ladungen erhält man einen Wert von $Q_{f L}=4,1 \cdot 10^{11} \mathrm{q} \mathrm{cm}^{-2}$.

Die Analyse der Leitwertmessungen ergibt drei gut auflösbare Niveaus von Oberflächenzuständen, für die folgende Werte ermittelt werden:

\begin{tabular}{llcl}
\hline Niveau & Energie $E_{t}-E_{v}$ & Relaxationszeit & Zustandsdichte \\
\hline I & $0,38 \mathrm{eV}$ & $1,7 \cdot 10^{-4} \mathrm{~s}$ & $8,9 \cdot 10^{10}(\mathrm{eV})^{-1} \mathrm{~cm}^{-2}$ \\
II & $0,59 \mathrm{eV}$ & $5,5 \cdot 10^{-4} \mathrm{~s}$ & $8,9 \cdot 10^{10}(\mathrm{eV})^{-1} \mathrm{~cm}^{-2}$ \\
III & $0,80 \mathrm{eV}$ & $>20 \cdot 10^{-4} \mathrm{~s}$ & - \\
\hline
\end{tabular}

Bei Bestrahlung mit Licht wird die Relaxationszeit dieser Zustände zum Teil wesentlich verringert. Aus der Wellenlängenabhängigkeit des Effektes schliessen wir, dass diese Verringerung ausschliesslich durch die erhöhte Konzentration von Minoritätsladungsträgern verursacht wird und die direkte optische Anregung dagegen keine Rolle spielt.

\title{
Untersuchung über den Charge-Pumping Effekt bei MOS-Transistoren
}

\author{
von J. Golder, E. Baldinger \\ (Institut für angewandte Physik der Universität Basel) \\ und W. Czaja \\ (RCA Laboratories, Zürich)
}

Bei Änderungen der Gatevorspannung erfolgt ein Umbesetzen von Zuständen an der Grenzschicht $\mathrm{Si}_{-} \mathrm{SiO}_{2}$. Die dazu erforderlichen Ladungsträger werden beim Enhancement-Mode MOS-Transistor einerseits von den Drain- und Source-Regionen und andererseits vom Substrat geliefert. Die analytische Beschreibung der Vorgänge basiert auf der Shockley-Read-Hall Statistik, welche für genügend kleine Variationen durch lineare Beziehungen approximiert werden kann. Bei grösseren Variationen bewirken die Abweichungen von der Linearität ein Überwiegen der Einfangsprozesse beim Umbesetzen der Zustände. Deshalb entsteht bei periodischen Variationen der Gatespannung, wie sie beim Charge-Pumping angewandt werden, ein nicht verschwindender Mittelwert des Substratstroms. Aus der Gegenüberstellung von Theorie und Experiment ergeben sich die Einfangsquerschnitte $\sigma_{n}$ und $\sigma_{p}$ der am Effekt beteiligten Zustände für Elektronen und Löcher.

\section{Untersuchung der Beweglichkeit von Ladungsträgern in GaSe}

von R. Minder, E. BALdinger und I. ZschoKKE-GränACHER

(Institut für angewandte Physik der Universität Basel)

In der Oberfläche eines Kristallplättchens erzeugt ionisierende Strahlung Elektron-Loch Paare, die in einem angelegten Feld getrennt werden können. Aus dem zeitlichen Verlauf des gemessenen Stroms erhält man Informationen über Beweglichkeit und Lebensdauer der Ladungsträger (Transient Charge Technique). Für die Experimente wurden GaSe-Plättchen mit aufgedampften Goldkontakten verwendet. 